\title{
Sensitivity to the Neutrino Mass Hierarchy of KM3NeT/ORCA
}

\author{
Martijn Jongen* for the KM3NeT collaboration \\ Nikhef, Amsterdam \\ E-mail: mjongen@nikhef.nl
}

$\mathrm{KM} 3 \mathrm{NeT}$ is the next-generation underwater neutrino telescope being installed in the Mediterranean Sea. Its low-energy branch KM3NeT/ORCA will measure neutrinos in the energy range of several $\mathrm{GeV}$, aiming to resolve the long-standing question of whether the neutrino mass hierarchy is normal or inverted by measuring matter-induced oscillation effects in atmospheric neutrinos.

In this contribution, the expected sensitivity of KM3NeT/ORCA for a measurement of the mass hierarchy and other oscillation parameters is discussed, based on input from the latest Monte Carlo simulations. A detailed overview is given of the sensitivity study, the various inputs and the log-likelihood ratio method employed. In particular, the various systematics taken into account in the study will be given attention.

The 34th International Cosmic Ray Conference,

30 July- 6 August, 2015

The Hague, The Netherlands

${ }^{*}$ Speaker. 


\section{Introduction}

$\mathrm{KM} 3 \mathrm{NeT}$ is the next-generation underwater neutrino telescope being installed in the Mediterranean Sea. It uses an extensive array of photomultiplier tubes (PMTs) to detect Cherenkov light from charged particles created in neutrino interactions at the single photon level.

The cornerstone of KM3NeT technology is the Digital Optical Module (DOM) [1], a spherical glass vessel housing 31 3" PMTs. The DOMs are arranged in strings of 18 DOMs each. In turn these strings are arranged in building blocks of 115 strings. This technology will be employed to measure neutrinos in two very different energy ranges. The high-energy program called KM3NeT/ARCA ("Astroparticle Research with Cosmics in the Abyss") will focus on the detection of high-energy astrophysical neutrinos [2], whereas the low-energy program called KM3NeT/ORCA ("Oscillation Research with Cosmics in the Abyss") aims to study the properties of neutrinos themselves by looking at the oscillation signature of neutrinos created in the atmosphere [3][4].

ORCA will consist of a single building block with dimensions decreased with respect to ARCA, in order to be sensitive to much lower neutrino energies of several GeV. The current layout has a vertical spacing of 6 metres between DOMs and an average distance of 20 metres between strings, corresponding to an instrumented volume of approximately $3.7 \times 10^{6} \mathrm{~m}^{3}$. However, the detector geometry is still being optimised, and the effect of a larger vertical separation is under study. The strings will be deployed near Toulon, France.

ORCA's main goal is to determine the neutrino mass hierarchy (NMH) through a high-statistics measurement of the upgoing atmospheric neutrino spectrum. When neutrinos traverse matter their normal ('vacuum') oscillation is modified due to coherent forward scattering of their electronneutrino component on the electrons. This effect is known as the Mikheyev-Smirnov-Wolfenstein (MSW) effect [5]. For atmospheric neutrinos traversing the Earth resonances occur for certain combinations of matter density and neutrino energy. These resonances occur for neutrinos in the normal hierarchy $(\mathrm{NH})$ and for antineutrinos in the inverted hierarchy (IH), allowing for an experimental determination of the NMH.

Other than neutrino energy and direction, the experiment has some sensitivity to the neutrino flavour. Muon-flavour charged-current (CC) neutrino interactions have a distinctive muon track which can be used to distinguish them from other $\mathrm{CC}$ events and from neutral current (NC) events. This leads to two different channels for the measurement: 'track' events (with a clear muon track) and 'cascade' events (everything else). Consequently, a particle identification algorithm has been developed, as well as dedicated reconstruction algorithms for tracks [6] and cascades [7].

Since the observable variations in the neutrino spectrum are small and vary rapidly as a function of energy and zenith angle, high statistics and an excellent detector resolution are essential to the measurement. This is illustrated in Figure 1.

\section{Simulation Chain}

To calculate ORCA's sensitivity to the mass hierarchy and oscillation parameters we use a simulation chain that models the neutrino propagation and the detector response. It yields the expected number of events in the two channels as a function of energy and zenith angle for a given operation time of the detector. The result depends on the values of various systematics (among which are the 


\begin{tabular}{|c|c|c|}
\hline Parameter & Mean & Width \\
\hline$\theta_{13}\left[^{\circ}\right]$ & 9 & 0.4 \\
$\theta_{12}\left[^{\circ}\right]$ & 34 & 1 \\
$\Delta m^{2}\left[10^{-5} \mathrm{eV}^{2}\right]$ & 7.6 & 0.2 \\
$\Delta M^{2}\left[10^{-3} \mathrm{eV}^{2}\right]$ & 2.4 & 0.05 \\
\hline
\end{tabular}

Table 1: The mean values and widths of the Gaussian parameter distributions used in the simulation. These values are compatible with recent global fits [12] [13].

neutrino oscillation parameters) that model the uncertainties in the detector and physics models. Here we describe the steps of the simulation chain and motivate their implementation.

The atmospheric flux is based on the Honda et al. tables for the Fréjus site without mountain at solar minimum [8]. The Earth's electron density profile is calculated from the Preliminary Reference Earth Model [9], assuming a 1:1 ratio between protons and neutrons throughout. The oscillation probabilities are calculated using a custom code based on the Ohlsson and Snellman Cayley-Hamilton approach [10]. The true oscillation parameter values are drawn from the Gaussian distributions described in table 1 . Note that $\theta_{23}$ and $\delta_{\mathrm{CP}}$ are not listed since we generate results for many true values of these parameters and quote the hierarchy sensitivity as a function of them.

The detector response modelling is based on extensive MC studies. The track and cascade reconstruction algorithms, particle ID and atmospheric muon rejection [11] have been simulated and their combined effect leads to effective masses, particle identification probabilities and energy and angle resolutions that are used in the sensitivity study. Dedicated resolutions are implemented for neutral current interactions and for the electron- and muon-flavour charged-current neutrino interactions.

More specifically, the energy responses are two-dimensional matrices that define a probability distribution of reconstructed neutrino energy as a function of true neutrino energy. Similarly, the angle responses are three-dimensional, yielding the distribution of the reconstructed zenith angle as a function of both true neutrino energy and zenith angle. The effective mass is implemented as a function of both energy and zenith angle to account for the zenith dependence induced by both the DOM up/down-asymmetry and the shape of the instrumented volume. The particle identification probabilities are modelled as being energy-dependent only. All these matrices are filled directly from MC simulations.

The effect of particle misidentification is taken into account: for each of the simulated events the reconstruction algorithm is chosen based on the outcome of the particle ID - not on the true flavour. This means that, for example, a fraction of the charged-current electron neutrino events is reconstructed by the track reconstruction algorithm.

Apart from neutral current events there is a background contribution of atmospheric muons that pass the rejection cut. These are included in the simulation as an energy- and zenith angledependent template from atmospheric muon simulations.

The resulting hierarchy signature, including all the steps described above, is shown in Figure 1 , and compared to the ideal (perfect reconstruction) distributions. 


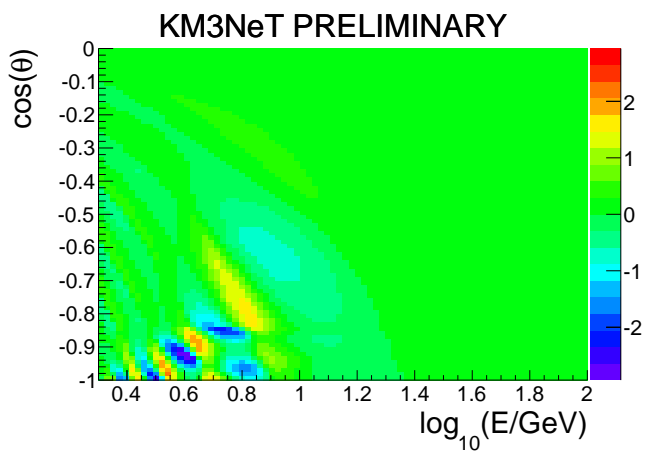

(a) Track channel (ideal).

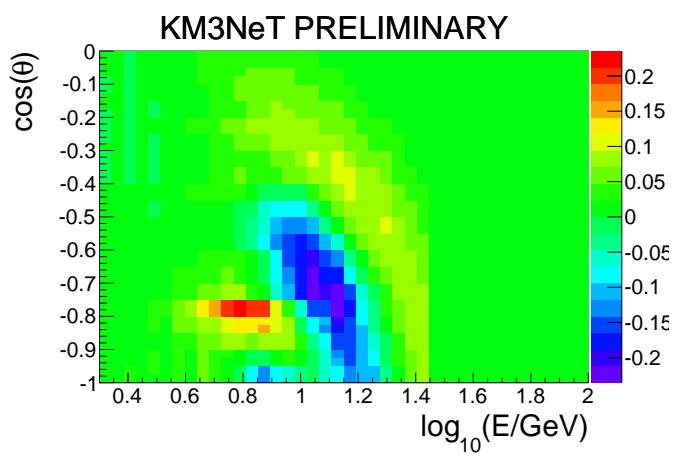

(c) Track channel (realistic).

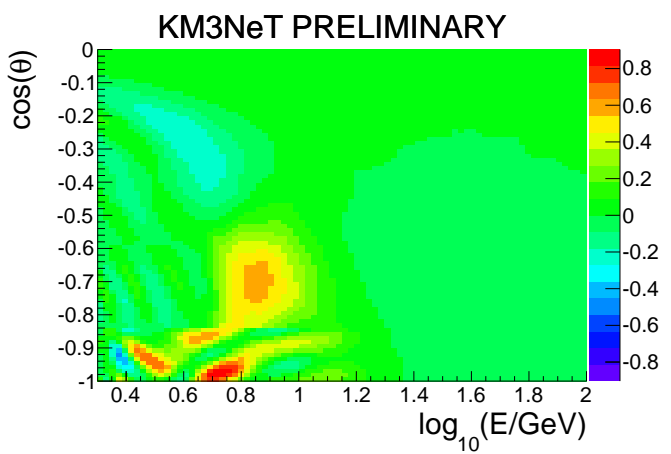

(b) Cascade channel (ideal).

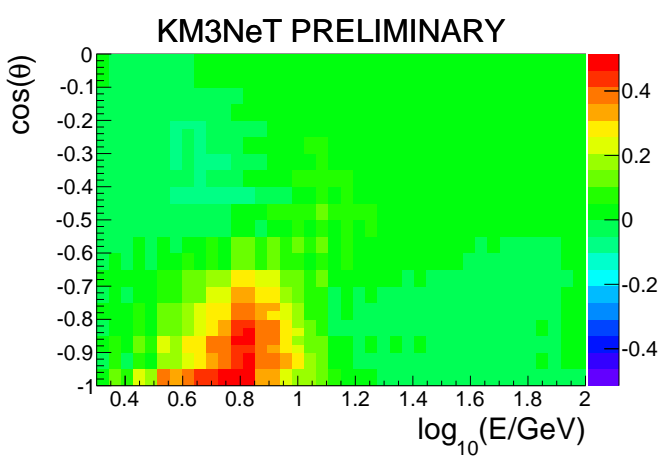

(d) Cascade channel (realistic).

Figure 1: The experimental signature of the mass hierarchy. Shown is the difference between the expected number of events given the $\mathrm{NH}$ and the $\mathrm{IH}$, divided by the square root of the number of events for the NH. Note that in this simplified approach all parameters other than the hierarchy are kept fixed. The top row shows an idealized case with perfect detector resolution (all other settings are unchanged); an intricate pattern is visible. In the bottom row the detector resolution has been folded in, smearing the pattern and leaving only a few large regions with an excess or deficit of expected events of $\mathrm{NH}$ compared to $\mathrm{IH}$.

\section{Sensitivity Calculation}

The expected sensitivity of ORCA to the neutrino mass hierarchy is calculated using a loglikelihood ratio (LLR) approach, which works as follows.

The simulation chain described in Sec. 2 is run for a given set of true values of the oscillation parameters and systematics, yielding expected event numbers. Based on the latter a Poissondistributed pseudo-experiment is drawn for which the best-fit parameter values are then found by maximizing the likelihood with respect to the parameters, once assuming $\mathrm{NH}$ and once assuming IH. The logarithm of the ratio between the two best-fit likelihood values (LLR) is used as the discriminating variable (test statistic) for the NMH.

The above procedure is repeated many times with the true values being sampled randomly from the global-fit-constrained parameter space. The separation between the resulting LLR distributions (see Figure 2) indicates how well NH can be distinguished from IH. The final significance number 


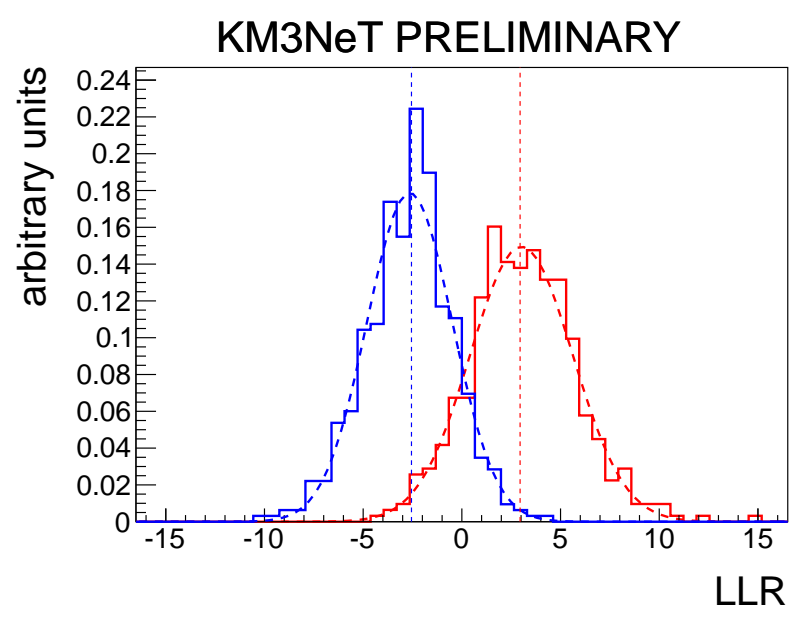

Figure 2: Typical log-likelihood ratio (LLR) distributions (solid lines), and Gaussian fits (dashed lines), for $\mathrm{NH}$ (red) and IH (blue). The dashed vertical lines mark the medians. This plot is for 3 years of operation time and $\theta_{23}=42^{\circ}$.

is the median significance deduced from those distributions. It is calculated from the fraction of events in the tail of the $\mathrm{NH}(\mathrm{IH})$ distribution that lies beyond the $\mathrm{IH}(\mathrm{NH})$ median. Gaussian fits are used to estimate the significance, since the distributions are sufficiently well-behaved.

The sensitivity to the oscillation parameters and systematics themselves can be deduced from the width of the best-fit-value distributions. Correlations among the degrees of freedom are also revealed due to the simultaneous fit.

\section{Systematics}

The NMH measurement relies on subdominant effects in the observed neutrino spectrum, therefore systematic effects that could alter the observed spectrum have to be studied in detail. Currently, the following systematics are included in the study: an overall factor in the number of events, which accounts for uncertainties in the absolute scaling of the atmospheric neutrino flux, the detector efficiency, and the neutrino-nucleon interaction cross-section; the ratio between neutrino and antineutrino events, defined such that the total number of events remains constant; the ratio between muon-flavour and electron-flavour events; a scaling of the neutral current contamination which accounts for the unknown absolute size of the NC interaction cross-section; and the slope of the atmospheric neutrino energy spectrum.

To evaluate the effect of the systematics on the mass hierarchy sensitivity, several simulations taking into account varying numbers of systematics were run and compared to a reference simulation that takes into account the oscillation parameters but none of the systematics (see Figure 3a).

In five dedicated simulations each of the systematics was added individually to the reference simulation, in order to gauge their effect in isolation. Additionally, a final simulation was run that took into account all systematics simultaneously (see Figure $3 b$ ). 


\begin{tabular}{|l|c|c|}
\hline systematic & individual uncertainty & combined uncertainty \\
\hline overall flux factor & $0.4 \%$ & $2.0 \%$ \\
$\nu / \bar{v}$ ratio & $2.1 \%$ & $4.0 \%$ \\
$\mu / e$ ratio & $0.8 \%$ & $1.2 \%$ \\
NC scaling & $2.9 \%$ & $11.0 \%$ \\
Energy slope & $0.2 \%$ & $0.5 \%$ \\
\hline
\end{tabular}

Table 2: The uncertainties (widths of the best-fit value distributions) for the five different systematics taken into account for this study. The second column shows the uncertainty when a single systematic is added to the reference simulation while the third column shows the uncertainty when all five are fitted simultaneously.

In all cases the three most important oscillation parameters - $\theta_{23}, \Delta M^{2}$ and $\delta_{\mathrm{CP}}$ - were fitted. The other oscillation parameters were treated as nuisance parameters by assigning random "best fit" values from the true value distributions to each pseudo-experiment, as it has been shown in earlier simulations that all of them either have negligible effects or are constrained sufficiently by other experiments. The true value of $\delta_{\mathrm{CP}}$ was drawn from a uniform distribution between 0 and $2 \pi$. The true values of the systematics were kept constant.

\section{Results}

The results from the reference simulation are shown in Figure $3 \mathrm{a}$. From this figure it is clear that the hierarchy significance depends strongly on the true value of $\theta_{23}$.

The fit of the systematics in each of the five dedicated simulations works very well without the need to add priors based on best-fit world values. The fit uncertainty for each systematic is shown in the second column of table 2. The effect of the individual systematics on the MHS is of the order of $10 \%$ or less at the two reference values $\theta_{23}=42^{\circ}$ and $\theta_{23}=48^{\circ}$. The current simulations are not yet precise enough to compare the effects of the individual systematics quantitatively. This will become possible as more pseudo-experiments are simulated.

Figure $3 \mathrm{~b}$ shows the result of the final simulation, including all five systematics. By comparing it to $3 \mathrm{a}$ we find that at $\theta_{23}=42^{\circ}$ the expected sensitivity after three years of operation time drops from $3.2 \sigma(3.9 \sigma)$ to $2.6 \sigma(2.2 \sigma)$ for $\mathrm{NH}(\mathrm{IH})$ due to the inclusion of systematic effects. At $48^{\circ}$ it drops from $7.1 \sigma(3.6 \sigma)$ to $5.3 \sigma(2.8 \sigma)$ for $\mathrm{NH}(\mathrm{IH})$.

It is important to note that the quoted numbers are averaged over the true value of $\delta_{\mathrm{CP}}$. This has a non-negligible effect on the measurement. As shown in Figure 4, the mass hierarchy measurement is most sensitive for a second-octant (i.e. $>45^{\circ}$ ) value of $\theta_{23}$ and no CP-violation. Fixing $\delta_{C P}$ to 0 (as is often done) is a best-case scenario assumption yielding a significantly higher expected $\mathrm{NMH}$ sensitivity. This is also illustrated by Figure 3c, where the true and 'fitted' value of $\delta_{\mathrm{CP}}$ have been fixed to 0 . There is an overall rise in MHS compared to $3 \mathrm{~b}$.

ORCA will also further constrain $\theta_{23}$ and the large mass-squared difference $\Delta M^{2} \equiv \frac{1}{2}\left(\Delta m_{32}^{2}+\right.$ $\left.\Delta m_{31}^{2}\right)$, improving considerably on the current knowledge of these parameters. The expected precision after three years at a reference value of $\theta_{23}=42^{\circ}$ is $3.8 \times 10^{-5} \mathrm{eV}^{2}$ for $\Delta M^{2}$ and $1.25^{\circ}$ for 


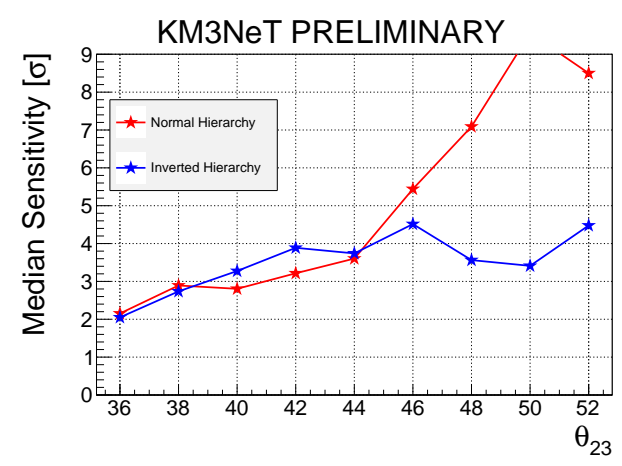

(a) Without systematics.

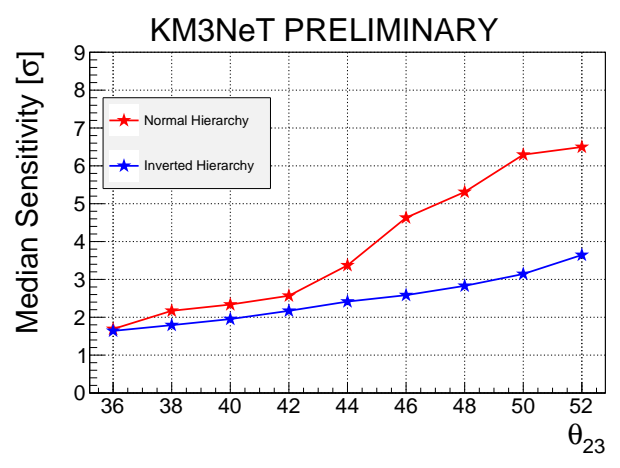

(b) With systematics.

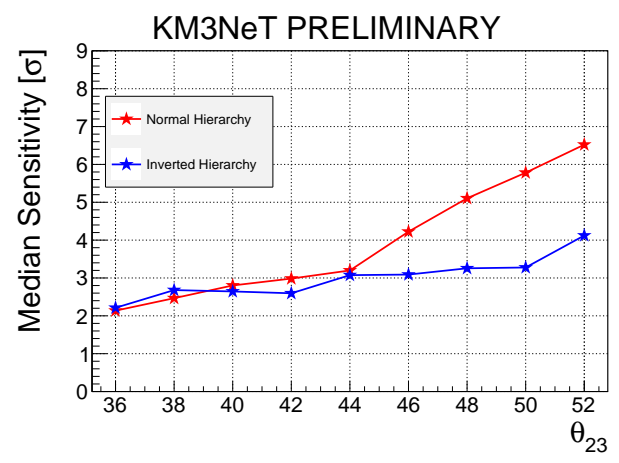

(c) With systematics, $\delta_{\mathrm{CP}}$ fixed to 0 .

Figure 3: The mass hierarchy sensitivity for 3 years of ORCA operation time as a function of $\theta_{23}$. The chosen range of $\theta_{23}$ roughly coincides with the global fit $3 \sigma$ range [13].

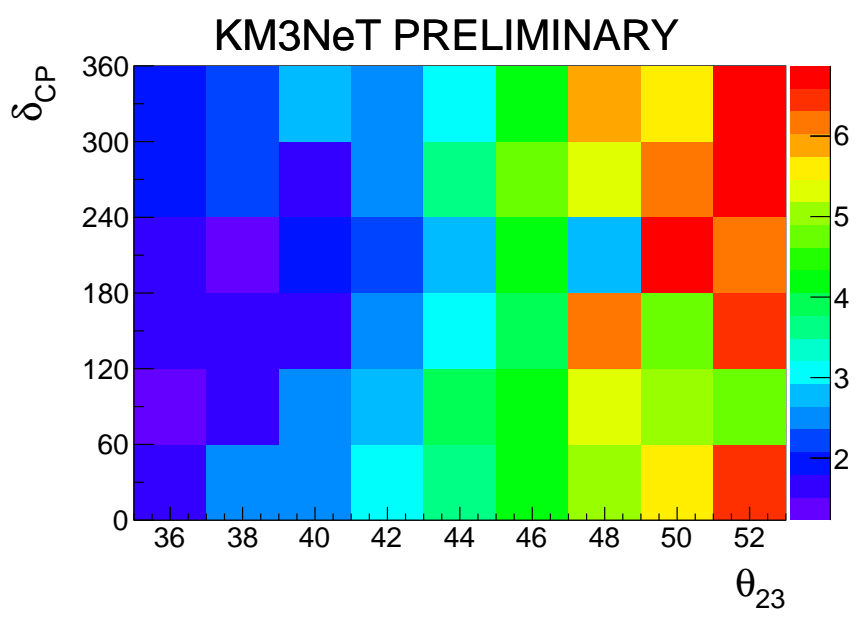

Figure 4: The NH mass hierarchy sensitivity in sigmas for 3 years of ORCA operation time as a function of $\theta_{23}$ and the CP-violating angle $\delta_{\mathrm{CP}}$. Note that the statistical uncertainty on the individual numbers is rather large, but sufficiently small to illustrate the general trend. This is for the full simulation including systematics. Note that the true value of $\theta_{23}$ is constant within each bin, whereas the true $\delta_{\mathrm{CP}}$ is drawn from a uniform distribution. 
$\theta_{23}$. These numbers are the widths of Gaussian fits of the best-fit-value distributions. Note that for $\theta_{23}$, only best-fit values in the first octant were included.

\section{Conclusions}

KM3NeT/ORCA will be a densely instrumented configuration of KM3NeT, designed to study the oscillation of atmospheric neutrinos at energies of order $10 \mathrm{GeV}$ in order to resolve the neutrino mass hierarchy.

A method to calculate the sensitivity of ORCA to the NMH has been presented, which takes into account five systematic parameters and fits to $\Delta M^{2}, \theta_{23}$ and $\delta_{\mathrm{CP}}$. Assuming NH, $\theta_{23}=42^{\circ}$, and fixing $\delta_{\mathrm{CP}}$ to 0 , ORCA is expected to determine the $\mathrm{NMH}$ with $3.0 \sigma$ significance within 3 years (Figure 3c), although the sensitivity is lower when a fit of $\delta_{\mathrm{CP}}$ is included. ORCA will also measure $\Delta M^{2}$ and $\theta_{23}$, with an expected resolution of $3.8 \times 10^{-5} \mathrm{eV}^{2}$ and $1.25^{\circ}$, respectively, in 3 years.

\section{References}

[1] R. Bruijn. These proceedings.

[2] P. Piattelli. These proceedings.

[3] J. Brunner. These proceedings.

[4] E. Akhmedov, S. Razzaque, A. Smirnov, JHEP 1302 (2013) 082.

[5] Wolfenstein L 1978 Phys. Rev. D17 2369

Mikheev S P and Smirnov A Yu 1985 Yad. Fiz. 421441 (Sov. J. Nucl. Phys. 42 913)

[6] S. Galata. These proceedings.

[7] J. Hofestädt. These proceedings.

[8] M. Honda et al. arXiv:1502.03916 [astro-ph.HE]

[9] A. Dziewonski, D. Anderson. Physics of the Earth and Planetary Interiors, Volume 25, Issue 4, June 1981, Pages 297-356, ISSN 0031-9201

[10] T. Ohlsson, H. Snellman. J.Math.Phys. 41 (2000) 2768-2788; Erratum-ibid. 42 (2001) 2345

[11] L. Fusco. These proceedings.

[12] F. Capozzi, G.L. Fogli et al. arXiv:1312.2878v2 [hep-ph]

[13] Forero, Tórtola, Valle. arXiv:1405.7540v3 [hep-ph] 